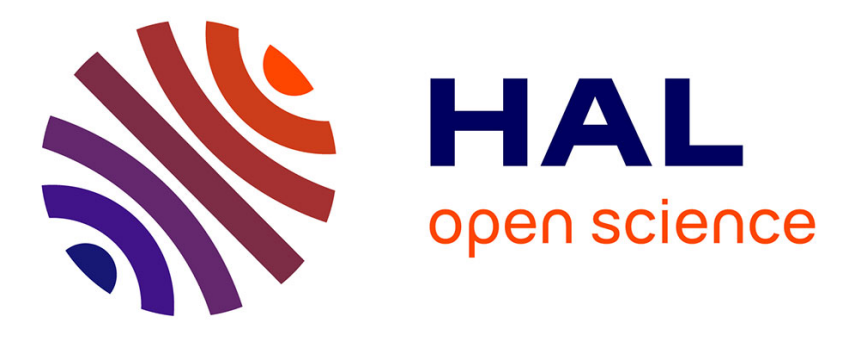

\title{
Nonlinear dynamics of circular capacitive micromachined ultrasonic transducers
}

Najib Kacem, Aymen Jallouli, Vincent Walter, Gilles Bourbon, Patrice Le Moal, Joseph Lardies

\section{- To cite this version:}

Najib Kacem, Aymen Jallouli, Vincent Walter, Gilles Bourbon, Patrice Le Moal, et al.. Nonlinear dynamics of circular capacitive micromachined ultrasonic transducers. Conference on Sensors, Nov 2015, Busan, South Korea. 10.1109/ICSENS.2015.7370489 . hal-03107840

\section{HAL Id: hal-03107840 \\ https://hal.science/hal-03107840}

Submitted on 12 Jan 2021

HAL is a multi-disciplinary open access archive for the deposit and dissemination of scientific research documents, whether they are published or not. The documents may come from teaching and research institutions in France or abroad, or from public or private research centers.
L'archive ouverte pluridisciplinaire HAL, est destinée au dépôt et à la diffusion de documents scientifiques de niveau recherche, publiés ou non, émanant des établissements d'enseignement et de recherche français ou étrangers, des laboratoires publics ou privés. 


\title{
Nonlinear Dynamics of Circular Capacitive Micromachined Ultrasonic Transducers
}

\author{
Najib Kacem, Aymen Jallouli, Vincent Walter, Gilles Bourbon, Patrice Lemoal and Joseph Lardies \\ FEMTO-ST Institute - UMR 6174, Applied Mechanics Department \\ 24 chemin de l'Épitaphe, 25000 Besançon, France \\ Email: najib.kacem@femto-st.fr
}

\begin{abstract}
A multiphysics model for capacitive micromachined ultrasonic transducers (CMUTs) is developed taking into account the main sources of nonlinearities in the case of circular plates. The equation of motion is reduced to an asymmetric Duffing equation which is solved using the harmonic balance method coupled with the asymptotic numerical method. Multiple-valued solutions, indicating the occurrence of jump phenomena, are observed numerically and confirmed experimentally on measured frequency responses of micro-machined CMUTs based on anodic bonding of a SOI wafer on a glass wafer. This purely numerical approach allows designers to control the resonator bifurcation topology by tuning the design parameters and thus gives the possibility of driving the CMUT beyond its critical amplitude. As a consequence, the CMUT performances can be enhanced in terms of bandwidth and generated acoustic power.
\end{abstract}

\section{INTRODUCTION}

Microelectromechanical resonators have been the subject of extensive research for several years and have generated much excitement as their use in commercial applications has increased. Due to the small size of these resonators, nonlinearities occur at very low amplitudes, which greatly reduce the useful linear dynamic range [1], [2]. Actually, it is a challenge to drive micro-resonators at large amplitudes without deteriorating their frequency stability.

Among these resonators, Capacitive Micromachined Ultrasonic Transducers (CMUTs) have recently attracted the attention of scientists and engineers. CMUTs are microelectromechanical devices which are used for the generation and sensing of acoustic signals in the ultrasonic range and they are composed of two micro plates where the bottom electrode is fixed while the top electrode is vibrating. The distance between the two electrodes is called gap. The vibration of the top electrode is due to the combination of the two electrostatic forces: a $D C$ voltage deflects the microplate downward, and an $A C$ voltage added to the bias voltage causing the vibration of the membrane which produces ultrasound waves.

CMUTs are used in many applications such as generating 2D and 3D ultrasound images [3], [4], nondestructive evaluation [5] and developing capacitive pressure sensors [6]. In order to enhance the performances of CMUTs in term of generated acoustic power, it is necessary to accurately model their dynamic behavior. Among several modeling techniques, the Finite Element Method (FEM) is the most used one which is mostly limited to the linear regime [7], [8], [9], [10], [11], while nonlinear models have been devoted to slightly nonlinear dynamics [12].
In this paper, a multiphysics model for the dynamics of circular CMUTs is developed, including Von Karman and electrostatic nonlinearities. The modal decomposition is used to transform the nonlinear partial differential equation into a finite degrees of freedom system which is numerically solved using the harmonic balance method (HBM) coupled with the asymptotic numerical method (ANM). A qualitative validation of the theoretical simulations with respect to experimental measurements is performed on a circular microplate electrostatically driven at large displacement with respect to the gap.

\section{DESIGN AND MODEL}

\section{A. Equations of motion}

The considered CMUT shown in Figure 1 consists of a clamped circular microplate actuated by a static electric force $V d c$ and an harmonic one $V a c$ at the frequency $\Omega$. Following Vogl and Nayfeh [12], the differential equation of circular, homogeneous and isotropic plate could be written as:

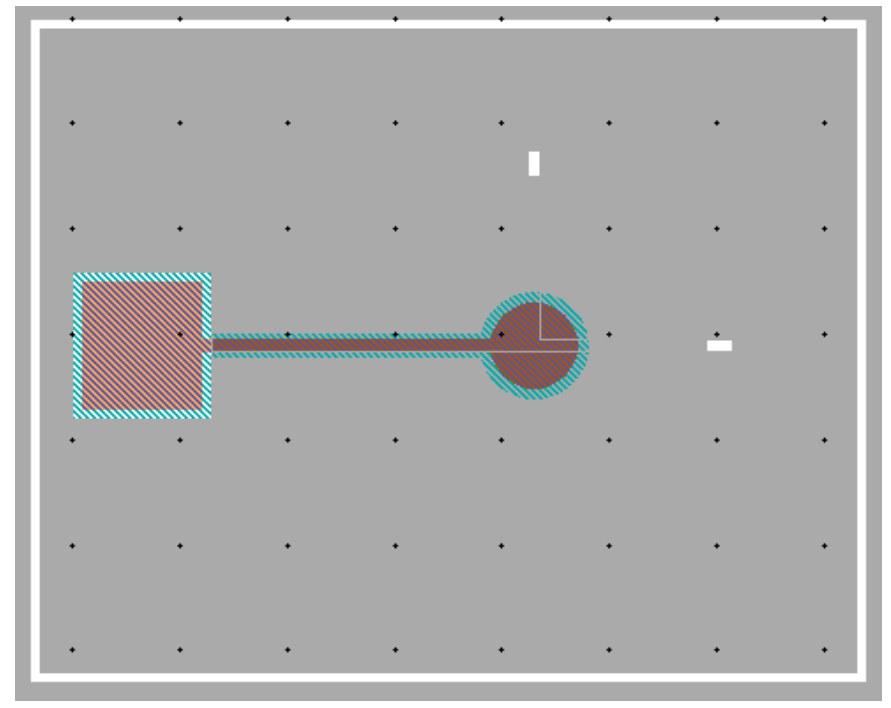

Fig. 1. An example of a CMUT design.

$$
\begin{gathered}
\rho h \frac{\partial^{2} \tilde{w}}{\partial \tilde{t}^{2}}+2 \tilde{c} \frac{\partial \tilde{w}}{\partial \tilde{t}}+D \tilde{\nabla}^{4} \tilde{w}=\frac{1}{\tilde{r}} \frac{\partial}{\partial \tilde{r}}\left(\frac{\partial \tilde{w}}{\partial \tilde{r}} \frac{\partial \tilde{\Phi}}{\partial \tilde{r}}\right) \\
+\tilde{\tau} h \tilde{\nabla}^{2} \tilde{w}+\tilde{F}+\frac{\varepsilon_{0} \tilde{v}^{2}(\tilde{t})}{2(d-\tilde{w})^{2}}
\end{gathered}
$$




$$
\tilde{\nabla}^{4} \tilde{\Phi}=-E h \frac{\partial^{2} \tilde{w}}{\partial \tilde{r}^{2}}\left(\frac{1}{\tilde{r}} \frac{\partial \tilde{w}}{\partial \tilde{r}}\right)
$$

and

$$
\tilde{\nabla}^{4}=\left(\frac{\partial^{2}}{\partial \tilde{r}^{2}}+\frac{1}{\tilde{r}} \frac{\partial}{\partial \tilde{r}}\right)^{2}
$$

The hat denotes a dimensional quantity, $\tilde{w}$ is the downward deflection, $\tilde{c}$ is the damping coefficient, $\rho$ is the material mass density, $h$ is the microplate thickness, $\tilde{\Phi}$ is the stress function, $E$ is Young's modulus, $D$ is the plate flexural rigidity defined as $D=\frac{E h^{3}}{12\left(1-\nu^{2}\right)}, \nu$ is the Poisson's ratio. The effective gap distance between the top and bottom electrodes is denoted by $d, \varepsilon_{0}$ is the electric permittivity of the gap medium between the plate and electrode, $\tilde{\tau}$ is the residual stress, $\tilde{F}$ is an additional downward pressure, and $\tilde{v}(\tilde{t})$ is the applied voltage. The boundary conditions of the vibrating circular plate are:

$$
\begin{array}{r}
\tilde{w}(R, \tilde{t})=0, \frac{\partial \tilde{w}(R, \tilde{t})}{\partial \tilde{r}}=0, \tilde{w}(0, \tilde{t}) \text { is bounded } \\
\frac{\partial^{2} \tilde{\Phi}(R, \tilde{t})}{\partial \tilde{r}^{2}}-\frac{\nu}{R} \frac{\partial \tilde{\Phi}(R, \tilde{t})}{\partial \tilde{r}}=0, \tilde{\Phi}(0, \tilde{t}) \text { is bounded }
\end{array}
$$

\section{B. Normalization}

For convenience and equation simplicity, we introduce the nondimensional variables:

$$
\begin{array}{cc}
\tilde{r}=R r, & \tilde{t}=R^{2}\left(\frac{\rho h}{D}\right)^{1 / 2} t, \\
\tilde{w}=d w, & \tilde{c}=\frac{(D \rho h)^{1 / 2}}{R^{2}} c, \\
\tilde{F}=\frac{D d}{R^{4}} F, & \tilde{v}^{2}(\tilde{t})=\frac{2 D d^{3}}{\varepsilon_{0} R^{4}} v^{2}(t), \\
\tilde{\tau}=\frac{D}{R^{2} h} \tau, & \tilde{\Phi}=E h d^{2} \Phi
\end{array}
$$

We transform the equation (1) and (3) into:

$$
\begin{aligned}
\frac{\partial^{2} w}{\partial t^{2}}+2 c \frac{\partial w}{\partial t}+\nabla^{4} w & =\frac{\beta}{r} \frac{\partial}{\partial r}\left(\frac{\partial w}{\partial r} \frac{\partial \Phi}{\partial r}\right)+\frac{\tau}{r} \frac{\partial}{\partial r}\left(r \frac{\partial w}{\partial r}\right) \\
+F(r, t)+\frac{v^{2}(t)}{(1-w)^{2}} & \\
\nabla^{4} \Phi & =-\frac{1}{r} \frac{\partial^{2} w}{\partial r^{2}}\left(\frac{\partial w}{\partial r}\right)
\end{aligned}
$$

where $\beta$ is a nondimensional parameter defined as:

$$
\beta=12\left(1-\nu^{2}\right) d^{2} / h^{2}
$$

The nondimensional boundary conditions of the system are:

$$
\begin{gathered}
w(1, t)=0, \frac{\partial w(1, t)}{\partial r}=0, w(0, t) \text { is bounded } \\
\frac{\partial^{2} \Phi(1, t)}{\partial r^{2}}-\nu \frac{\partial \Phi(1, t)}{\partial r}=0, \Phi(0, t) \text { is bounded }
\end{gathered}
$$

\section{Reduced order model}

A reduced-order model is generated by modal decomposition transforming Equations (7) and (8) into a finite degreeof-freedom system consisting of ordinary differential equations in time. We use the undamped linear mode shapes of the membrane as basis functions in the Galerkin procedure. To this end, we express the deflection as

$$
w(r, t)=\sum_{m=1}^{N} \eta_{m}(t) \phi_{m}(r)
$$

where $\phi_{m}(r)$ is the $m^{t h}$ shape function, $\eta_{m}(t)$ is the $m^{t h}$ generalized coordinate, and $N$ is the number of retained modes. Due to the symmetry of the problem, only the symmetric modes are considered. Following Nayfeh [13], the shape functions for a clamped circular plate actuated by symmetric forces can be written as:

$$
\phi_{m}(r)=\frac{J_{0}\left(r \sqrt{\Omega_{\mathrm{m}}}\right)}{J_{0}\left(\sqrt{\Omega_{\mathrm{m}}}\right)}-\frac{I_{0}\left(r \sqrt{\Omega_{\mathrm{m}}}\right)}{I_{0}\left(\sqrt{\Omega_{\mathrm{m}}}\right)}
$$

where $J_{0}$ and $I_{0}$ are respectively the first kind Bessel function and the modified Bessel function and $\Omega_{m}$ is the nondimensional resonance frequency for $\phi_{m}(r)$. The mode shapes are chosen orthonormal:

$$
\int_{0}^{1} r \phi_{m}(r) \phi_{n}(r) d r=\delta_{m n}
$$

Substituting Equation (12) into Equations (7) and (8), multiplying by $(1-w)^{2} r \phi_{q}(r)$ and integrating the resulting equation over $r \in[0,1]$, we obtain the following nonlinear system:

$$
M(\eta) \ddot{\eta}+2 c M(\eta) \dot{\eta}+N(\eta) \eta=P(\eta)+v^{2}(t) L
$$

where $M(\eta)$ and $N(\eta)$ are $N \times N$ nonlinear matrices and $P(\eta)$ is a $N \times 1$ nonlinear vector, $\eta(t)=$ $\left\{\eta_{1}(t), \eta_{2}(t), \ldots, \eta_{N}(t)\right\}$ is a $N \times 1$ vector containing the generalized coordinates.

\section{NUMERICAL SimUlations}

Vogl and Nayfeh [12] used the multiple scale method to analyze the CMUT primary resonance associated to the first bending mode. Nevertheless, the obtained solutions are only valid for a low level of nonlinearity. Therefore, we propose a computational solving procedure based on the harmonic balance method (HBM) coupled with the asymptotic numerical method (ANM), for which the solutions are valid up to very large displacement compared to the gap. This technique, proposed by Cochelin and Vergez [14], gives the periodic solutions of a dynamical system when a control parameter is varied. This solving procedure has been used successfully for the nonlinear equations of motion of micro-beams resonators [15] and Carbone nanotubes [16] under primary resonance. Once the system is transformed into a quadratic form, the HBM is used to obtain the associated nonlinear algebraic system which is solved using the ANM. As a first step, Equation (15) is solved for $N=1$.

Figure 2 shows the frequency responses computed numerically for a specific set of design parameters (diameter $=$ $160 \mu \mathrm{m}$, thickness $=2 \mu \mathrm{m}$, gap $=750 \mathrm{~nm}$ ) and several $D C$ voltages. Remarkably, the resonance peaks of the membrane moves towards low frequencies due to the linear negative stiffness proportional to $V d c^{2}$. Beyond the CMUT critical amplitude [17], [18], a nonlinear spring softening effect is also notable for $V d c=65 \mathrm{~V}$ and a high $A C$ voltage $(\mathrm{Vac}=10 \mathrm{~V})$ which is induced by the increase of the Duffing stiffness and the amplitude of the excitation force. 


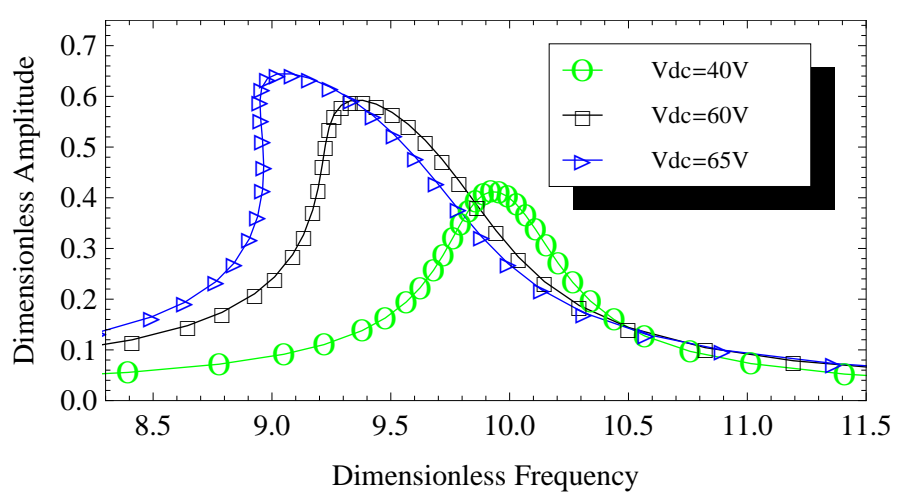

Fig. 2. Theoretical frequency responses of the designed CMUT for $V a c=$ $10 \mathrm{~V}$ and several $D C$ voltages.

\section{FABRICATION}

Several CMUTs have been fabricated (Figures 3 and 4) in order to validate the nonlinear phenomena captured using the numerical model. The process flow involves three main steps which are the etching of the cavities and evaporation of the bottom electrodes on the glass wafer, the anodic bonding of the glass wafer on the SOI wafer, and the etching of the handle layer and the buried oxide layer of the SOI wafer [19].

The glass wafer is a $544 \mu \mathrm{m}$ thick glass SCHOTT BOROFLOAT33 substrate since Borofloat33 thermal expansion coefficient $\left(\approx 3.25 \cdot 10^{-6} K^{-1}\right)$ is close to the silicon's one $\left(\approx 2.6 \cdot 10^{-6} K^{-1}\right)$. The cavity etching step defines both the membrane radius and the gap height. The first photolithography step defines the cavity shape on the glass Borofloat substrate. The glass is etched in a buffered hydrofluoric acid solution through the photoresist. The ratio of ammonium fluoride (NH4F) solution (NH4F 40\%) to hydrofluoric acid solution (HF 50\%) in this buffered hydrofluoric acid solution is 7:1. Finally, the first photoresist is removed to leave $1 \mu \mathrm{m}$ deep cavities.

The bottom electrodes are fabricated by lift off technology. The second photoresist is coated and patterned on the floor of the cavities and a $250 \mathrm{~nm}$ titanium-gold layer is evaporated. The second photoresist is removed before performing bonding, leaving the bottom electrodes in the cavities. The bottom electrodes are not electrically isolated from the conductive membrane. Therefore, the $D C$ voltage applied to the membrane has to be controlled very precisely to avoid electrical shorting. The cavity depth and the electrode thickness result in an electrostatic gap around $0.75 \mu \mathrm{m}$.

The membrane thickness is defined by the $2 \mu \mathrm{m}$ silicon device layer of the SOI wafer. Both SOI wafer and processed Pyrex 7740 wafer are cleaned and surface activated before anodic bonding. The contact is operated at ambient temperature, and then the coupled wafers are heated to $350^{\circ} \mathrm{C}$, previously to the electrical bonding procedure. The release of the membranes is achieved by removing the handle layer using potassium hydroxide $(\mathrm{KOH})$ wet etching and then the buried oxide layer of the SOI wafer using the buffered hydrofluoric acid (BHF) wet etching previously mentioned. The buried oxide layer first protects the silicon membrane from $\mathrm{KOH}$ solution before being removed in the buffered hydrofluoric acid solution. The
Silicon Membrane, $2 \mu \mathrm{m}$

Aluminum top electrode, $220 \mathrm{~nm}$

Titanium/Gold bottom electrode, $250 \mathrm{~nm}$

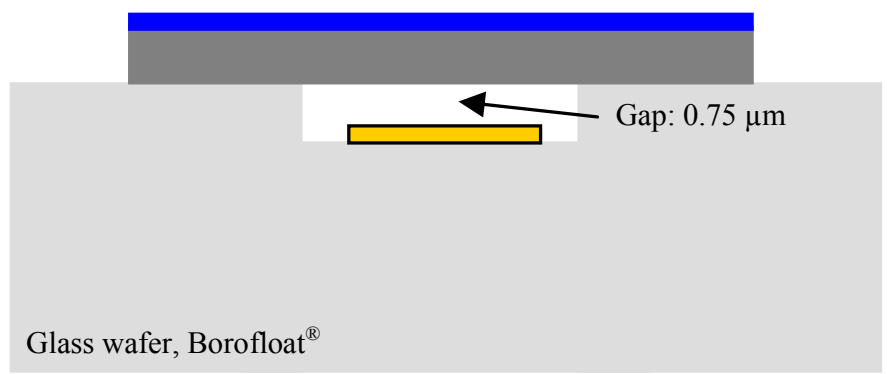

Fig. 3. A sectional side view of the designed CMUT.

Aluminum top electrode is deposited and wet etched in the third photoresist level. The last step is a silicon dry etching, using aluminum as a hard mask, to get access to the pad.

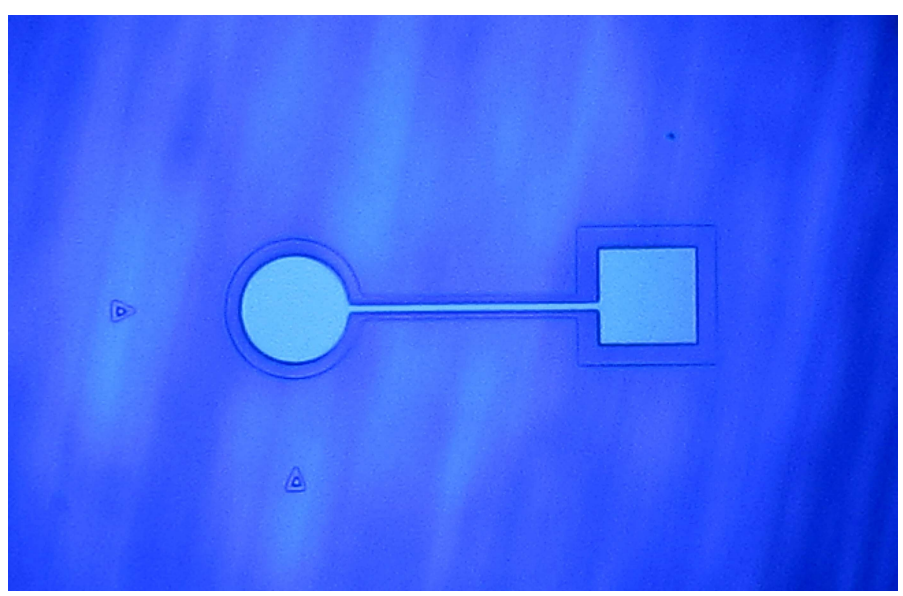

Fig. 4. An optical microscopy image of a fabricated CMUT.

\section{ElectromechanicAl CHARACTERIZATION}

The CMUT defined by a $80 \mu m$ radius, for a $2 \mu m$ thick membrane and a $0.75 \mu \mathrm{m}$ gap height is actuated with $V a c=$ $10 \mathrm{~V}$ and a high $D C$ voltage in order to reach large displacements below pull-in instability [20]. Unit cells are perfectly decoupled from the surrounding structures by wide interspaces. The CMUT characterization is mainly made on unit cells, i.e. on single membranes. The mechanical resonance frequency in air is measured. In the experimental procedure, the evolution of the resonance frequency is measured as a function of the $D C$ voltage using a POLYTEC laser vibrometer. The vibrometer is composed of a fiber-coupled vibrometer sensor head OFV-534 and an OFV-5000 controller. The controller has a VD-02 decoder, being able to measure displacements up to $1 \mu \mathrm{m}$ peak at a maximum of $1.5 \mathrm{MHz}$, with a resolution of $2.5 \mu \mathrm{ms}^{-1} / \sqrt{\mathrm{Hz}}$. It can be reminded that vibration amplitude is maximized at the mechanical resonance frequencies. 
Experimental results shown in Figure 5 are in good agreement with the numerical simulations. They confirm the effect of the $D C$ voltage in shifting the resonance frequency and giving rise to a nonlinear softening effect and bistability for $V d c=65 V$. This can be explained by the presence of strong electrostatic nonlinearities compared to geometric nonlinearities.

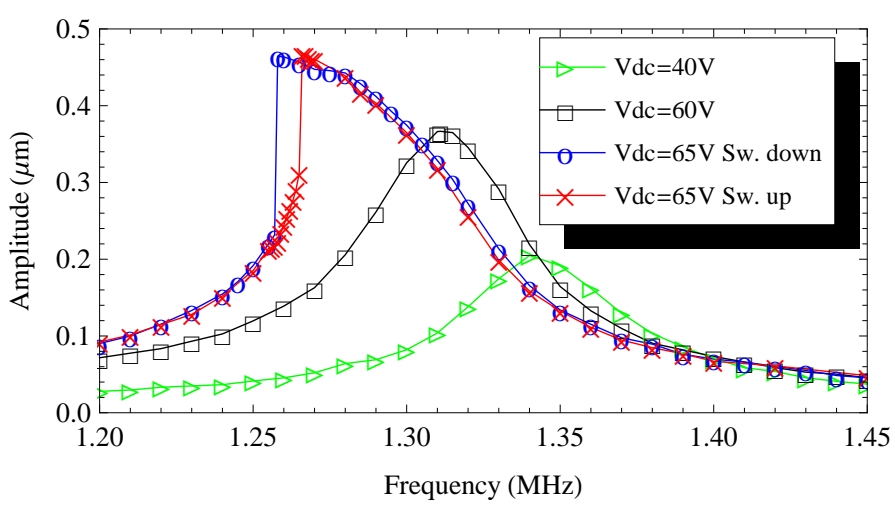

Fig. 5. Measured frequency responses of the fabricated CMUT for $V a c=$ $10 \mathrm{~V}$ and several $D C$ voltages.

\section{CONCLUSION}

In this paper, the nonlinear dynamics of circular CMUTs under primary resonance was investigated. The mathematical model, that takes into account the electrostatic and mechanical nonlinearities, is transformed into a set of coupled nonlinear ordinary differential equations using the Galerkin procedure. The harmonic balance method was then used to decompose the obtained system into a quadratic one which has been solved using the asymptotic numerical method. It has been shown that the $D C$ voltage has a direct impact on the resonance frequency and the hardening or softening nonlinear effects while $V a c$ affects mostly the shape of the resonance curves. The experimental validation of the model has been performed on CMUTS fabricated using anodic bonding of a SOI wafer on a glass wafer. The electromechanical characterization of a designed CMUT has been achieved using a laser vibrometer and comparisons between numerical and experimental results displayed an excellent agreement in resonance frequency, peak shape and amplitude. Hence, it proves the efficiency of the model as a predictive tool. In practice, the developed computational model can be used by MEMS designers to improve the performances of CMUTs in many applications such as 2D and $3 \mathrm{D}$ ultrasound images.

\section{ACKNOWLEDGMENT}

This project has been performed in cooperation with the Labex ACTION program (contract ANR-11-LABX-01-01). This work was partly supported by the french RENATECH network and its FEMTO-ST technological facility.

\section{REFERENCES}

[1] N. Kacem and S. Hentz, "Bifurcation topology tuning of a mixed behavior in nonlinear micromechanical resonators," Applied Physics Letters, vol. 95, no. 18, p. 183104, 2009.
[2] N. Kacem, S. Baguet, R. Dufour, and S. Hentz, "Stability control of nonlinear micromechanical resonators under simultaneous primary and superharmonic resonances," Applied Physics Letters, vol. 98, no. 19, pp.,- 2011.

[3] T. G. Fisher, T. J. Hall, S. Panda, M. S. Richards, P. E. Barbone, J. Jiang, J. Resnick, and S. Barnes, "Volumetric elasticity imaging with a $2 \mathrm{D}$ CMUT array," Ultrasound in medicine \& biology, vol. 36, no. 6, pp. 978-990, 2010.

[4] J. Song, S. Jung, Y. Kim, K. Cho, B. Kim, S. Lee, J. Na, I. Yang, O.k. Kwon, and D. Kim, "Reconfigurable 2D cMUT-asic arrays for 3D ultrasound image," in SPIE Medical Imaging. International Society for Optics and Photonics, 2012, pp. 83 201A-83 201A.

[5] X. Wang, Y. Fan, W.-C. Tian, H.-J. Kwon, S. Kennerly, G. Claydon, and A. May, "An air-coupled capacitive micromachined ultrasound transducer for noncontact nondestructive evaluation," in Sensors, 2007 IEEE. IEEE, 2007, pp. 1464-1467.

[6] H. Dudaicevs, M. Kandler, Y. Manoli, W. Mokwa, and E. Spiegel, "Surface micromachined pressure sensors with integrated CMOS readout electronics," Sensors and Actuators A: Physical, vol. 43, no. 1, pp. 157-163, 1994.

[7] B. Bayram, G. G. Yaralioglu, A. S. Ergun, and B. Khuri-Yakub, "Influence of the electrode size and location on the performance of a CMUT [us transducer]," in Ultrasonics Symposium, 2001 IEEE, vol. 2. IEEE, 2001, pp. 949-952.

[8] B. Bayram, E. Hæggstrom, G. G. Yaralioglu, and B. T. Khuri-Yakub, "A new regime for operating capacitive micromachined ultrasonic transducers," Ultrasonics, Ferroelectrics, and Frequency Control, IEEE Transactions on, vol. 50, no. 9, pp. 1184-1190, 2003.

[9] A. Lohfink and P.-C. Eccardt, "Linear and nonlinear equivalent circuit modeling of CMUTs," Ultrasonics, Ferroelectrics, and Frequency Control, IEEE Transactions on, vol. 52, no. 12, pp. 2163-2172, 2005.

[10] W. P. Mason, Electromechanical transducers and wave filters. D. Van Nostrand Co., 1948.

[11] O. Ahrens, A. Buhrdorf, D. Hohlfeld, L. Tebje, and J. Binder, "Fabrication of gap-optimized CMUT," Ultrasonics, Ferroelectrics, and Frequency Control, IEEE Transactions on, vol. 49, no. 9, pp. 13211329, 2002.

[12] G. W. Vogl and A. H. Nayfeh, "Primary resonance excitation of electrically actuated clamped circular plates," Nonlinear Dynamics, vol. 47, no. 1-3, pp. 181-192, 2007.

[13] A. H. Nayfeh and P. F. Pai, Linear and nonlinear structural mechanics. John Wiley \& Sons, 2008.

[14] B. Cochelin and C. Vergez, "A high order purely frequency-based harmonic balance formulation for continuation of periodic solutions," Journal of sound and vibration, vol. 324, no. 1, pp. 243-262, 2009.

[15] N. Kacem, S. Baguet, S. Hentz, and R. Dufour, "Computational and quasi-analytical models for non-linear vibrations of resonant MEMS and NEMS sensors," International Journal of Non-Linear Mechanics, vol. 46 , no. 3 , pp. $532-542,2011$

[16] S. Souayeh and N. Kacem, "Computational models for large amplitude nonlinear vibrations of electrostatically actuated carbon nanotube-based mass sensors," Sensors and Actuators A: Physical, vol. 208, pp. 10 20, 2014.

[17] N. Kacem, S. Hentz, D. Pinto, B. Reig, and V. Nguyen, "Nonlinear dynamics of nanomechanical beam resonators: improving the performance of NEMS-based sensors," Nanotechnology, vol. 20, no. 27, p. 275501, 2009.

[18] N. Kacem, J. Arcamone, F. Perez-Murano, and S. Hentz, "Dynamic range enhancement of nonlinear nanomechanical resonant cantilevers for highly sensitive NEMS gas/mass sensor applications," Journal of Micromechanics and Microengineering, vol. 20, no. 4, p. 045023, 2010.

[19] M. Bellaredj, G. Bourbon, V. Walter, P. L. Moal, and M. Berthillier, "Anodic bonding using SOI wafer for fabrication of capacitive micromachined ultrasonic transducers," Journal of Micromechanics and Microengineering, vol. 24, no. 2, p. 025009, 2014.

[20] N. Kacem, S. Baguet, S. Hentz, and R. Dufour, "Pull-in retarding in nonlinear nanoelectromechanical resonators under superharmonic excitation," Journal of Computational and Nonlinear Dynamics, vol. 7, no. 2, p. 021011, 2012. 